\title{
Generalized Stability for a Class of Nonlinear Difference Population Model
}

\author{
Özlem Ak Gümüş \\ Adıyaman Üniversitesi, Fen Edebiyat Fakültesi, Matematik Bölümü, Adıyaman \\ e-mail:akgumus@adiyaman.edu.tr
}

geliş tarihi; 01.04.2016 kabul tarihi; 21.11.2016

\section{Keywords}

Nonlinear Dynamic Models;

Stability;

Population Models;

Schur-Cohn Criteria;

Difference Equations;

Time Delay.

\section{Abstract}

In this study, non-linear difference population model has been considered. Stability conditions obtained by linearizing this model have been generalized to a certain step by presenting a different approach.

\section{Lineer Olmayan Fark Popülasyon Modelinin bir Sınıfı için Genelleştirilmiş Kararlılık}

\section{Anahtar kelimeler}

Lineer olmayan dinamik modeller;

Kararlilık;

Popülasyon Modelleri;

Schur-Cohn Kriteri;

Fark Denklemleri;

Zaman Gecikmesi;

Özet

Bu çalışmada, lineer olmayan fark popülasyon modeli gözönüne alındı. Bu model lineerleştirilerek elde edilen kararlılık şartları, farklı bir yaklaşım sunarak belirli bir adıma kadar genelleştirildi.

(c) Afyon Kocatepe Üniversitesi

\section{Introduction}

Life of organisms on earth, natural phenomena and problems encountered can be expressed with mathematical terms. This form of operation, which serves for expressing actual phenomena, is mathematical modeling. Phenomena connected to discrete-time variables in this way are given with difference models. Accordingly, non-linear model approach instead of simple configurations enables us to get better results. Recently, the subject of what the organism population will be in the future has become more salient. Consequently, what the conditions, which ensure the model to be stable, is important [see Merdan and Ak Gümüş (2012), Çelik et al (2008), Merdan and Karaoğlu (2012), Ak Gümüş (2014), Ak Gümüş and Köse (2012)]. Thus, we might have a precise knowledge on the number of living beings in a future time. Our aim is to provide a generalization of stability conditions of non-linear delayed difference equation given below for $\tau=K$, $K=1,2,3,4,5,6,7,8$

$$
N_{t+1}=\mu N_{t} f\left(N_{t-\tau}\right), \quad \lambda>0
$$

where $\mu$ is per capita growth rate, $N_{t}$ represents the population density at time $t, \tau$ is the time for sexual maturity. Also, $f\left(N_{t-\tau}\right)$ is the function describing interactions (competitions) among the mature individuals. The Eq.(1) is a single-species population model formed with a realistic approach. Stability examination of the model was conducted for $\tau=1 \quad$ [see Çelik et al (2008)]. Again, stability 
conditions for $\tau=2$ and $\tau=3$ were investigated [see Merdan and Ak Gümüş (2012), Merdan and Karaoğlu (2012)].

In this study, section 2 contains a different approach based on finding stability conditions for $\tau=K, \quad K=1,2,3,4,5,6,7,8$ in the Eq.(1). In section 3 , the examples which confirm the results are given. Section 4 was allocated to the conclusion part.

\section{Stability Conditions}

Definition 1. [Allen (2007)] For the first order difference equation $x_{t+1}=f\left(x_{t}\right)$ an equilibrium solution is a constant solution $\bar{x}$ to the difference equation, that is, a solution $\bar{x}$ satisfying

$$
\bar{x}=f(\bar{x}) \text {. }
$$

For the first order system,

$$
X_{t+1}=F\left(X_{t}\right)
$$

an equilibrium solution is a constant solution $\bar{X}$ satisfying

$$
\bar{X}=F(\bar{X}) \text {. }
$$

Local stability criteria for first order non-linear systems or for higher order non-linear difference equations depend on the behavior of the linearized system. Consider a first order system consisting of $n$ equations, $X(t)=\left(x_{1}(t), x_{2}(t), \ldots, x_{n}(t)\right)^{T}$,

$$
X_{t+1}=F\left(X_{t}\right)
$$

where $\quad F=\left(f_{1}, f_{2}, \ldots, f_{n}\right)^{T}$ and $f_{i} \equiv f_{i}\left(x_{1}, x_{2, \ldots}, x_{n}\right)$, $i=1,2, \ldots, n$. Suppose system (2) has an equilibrium at $\bar{X}$. Then if $U_{t}=X_{t}-\bar{X}$, linearization of system (2) about $\bar{X}$ leads to the system

$$
U_{t+1}=J U_{t},
$$

where $J$ is the Jacobian matrix evaluated at $\bar{X}$,

$$
J=\left(\begin{array}{cccc}
\frac{\partial f_{1}(\bar{X})}{\partial x_{1}} & \frac{\partial f_{1}(\bar{X})}{\partial x_{2}} & \cdots & \frac{\partial f_{1}(\bar{X})}{\partial x_{n}} \\
\frac{\partial f_{2}(\bar{X})}{\partial x_{1}} & \frac{\partial f_{2}(\bar{X})}{\partial x_{2}} & \cdots & \frac{\partial f_{2}(\bar{X})}{\partial x_{n}} \\
\vdots & \vdots & \cdots & \vdots \\
\frac{\partial f_{n}(\bar{X})}{\partial x_{1}} & \frac{\partial f_{n}(\bar{X})}{\partial x_{2}} & \cdots & \frac{\partial f_{n}(\bar{X})}{\partial x_{n}}
\end{array}\right) .
$$

Local asymptotic stability of $\bar{X}$ (or the system of containing $\bar{X}$, depends on the eigenvalues of the Jacobian matrix, which in turn depend on the existence of the partial derivatives in a region containing $\bar{X}$. Therefore, for local asymptotic stability of $\bar{X}$, we require that the partial derivatives of $F_{i}$ be continuous in an open set containing $\bar{X}$ [see Allen (2007)]. Equivalently, the solution of Eq.(2) is asymptotically stable if and only if $|\sigma|<1$ is held for every zero $\sigma$ of the characteristic polynomial of Jacobian matrix.

The equation obtained by linearizing the Eq.(1) for $\tau=K$ is in the form of

$$
x_{K+1}-x_{K}-p=0 \text {, }
$$

its characteristic polynomial

$$
g(\lambda)=\lambda^{K+1}-\lambda^{K}-p
$$

where, $\quad p=\mu f^{\prime}\left(N^{*}\right) N^{*}$ such that $f$ has a continuous second-order derivative in some interval $I$ containing $N^{*}$. Also, $N^{*}$ is the equilibrium point of the Eq.(1). First, let's give the relevant theorem for this.

Theorem 2 .(Schur-Cohn Criteria): The zeros of the characteristic polynomial (4) lie inside the unit disk if and only if the following hold:

$G(\sigma)=\sigma^{(k)}+p_{1} \sigma^{(k-1)}+p_{2} \sigma^{(k-2)}+\cdots+p_{k-2} \sigma^{2}+p_{k-1} \sigma+p_{k}(4)$

(i) $G(1)>0$,

(ii) $(-1)^{k} G(-1)>0$,

(iii) the $(k-1) \times(k-1)$ matrices 


$$
B_{k-1}^{ \pm}=\left[\begin{array}{rrrrr}
1 & 0 & \cdots & 0 & 0 \\
p_{1} & 1 & \cdots & 0 & 0 \\
\vdots & \vdots & \ddots & \vdots & \vdots \\
p_{k-3} & p_{k-2} & \cdots & 1 & 0 \\
p_{k-2} & p_{k-3} & \cdots & p_{1} & 1
\end{array}\right] \pm\left[\begin{array}{ccccc}
0 & 0 & \cdots & 0 & p_{k} \\
0 & 0 & \cdots & p_{k} & p_{k-1} \\
\vdots & \vdots & \ddots & \vdots & \vdots \\
0 & p_{k} & \cdots & p_{4} & p_{3} \\
p_{k} & p_{k-1} & \cdots & p_{3} & p_{2}
\end{array}\right]
$$

are positive innerwise [Elaydi (2006)].

Remark 3. The necessary and sufficient condition for the Eq.(1) to be stable, the Eq.(3) to be stable is for $\tau=K$.

Accordingly, considering the first two conditions from Theorem 2, the necessary and sufficient condition for the Eq.(3) to be stable is

$$
p<0
$$

and

$$
(-1)^{K+1}\left[(-1)^{K+1}-(-1)^{K}-p\right]>0 .
$$

If these conditions are considered, the cases $p<0$ and $p>-2$ are respectively obtained for odd and even values of $K$ in the Eq.(6). Then, let's give stability conditions for odd and even values of $K$ via the condition iii) from Theorem 2.

\subsection{Stability analysis of the equation (1) for odd number values of $K$}

Now, let's perform stability analysis for odd number values of $K$ in the Eq.(1). As per Theorem 2, each stability condition at $k$ steps should also added by the cases of positive absolute values of stability conditions from $k=1$ to that step. When the positive innerwise for conditions iii) in Theorem 2 are calculated by using Maple software for odd values of $K=2 k-1$, $k=1,2,3,4$, the stability conditions corresponding characteristic polynomials of $\lambda^{2 k}-\lambda^{2 k-1}-p$ of linearized equation, as follows: for $k=1$,

$$
\lambda^{2}-\lambda-p, \quad|p|<
$$

for $k=2$,

$$
\lambda^{4}-\lambda^{3}-p, \mid p 3-2 p<4 \quad p
$$

for $k=3$,

$$
\lambda^{6}-\lambda^{5}-p, \quad\left|p^{5}-4 p^{3}+3 p\right|<1-3 p^{2}+p^{4}
$$

for $k=4$,

$$
\lambda^{8}-\lambda^{7}-p, \quad\left|p^{7}-6 p^{5}+10 p^{3}-4 p\right|<1-6 p^{2}+5 p^{4}-p^{6}
$$

can be obtained. Additionaly, if the right side of the inequalities above are considered, coefficients of the terms $p^{i}, i=2,4,6$ are in the form of

Table 1

\begin{tabular}{llll}
\hline$\tau_{k}=\tau$ & $p^{2}$ & $p^{4}$ & $p^{6}$ \\
\hline$\tau_{2}=3$ & -1 & & \\
$\tau_{3}=5$ & -3 & 1 & \\
$\tau_{4}=7$ & -6 & 5 & -1 \\
$\tau_{5}=9$ & -10 & 15 & -7 \\
$\tau_{6}=11$ & -15 & 35 & -28 \\
$\tau_{7}=13$ & -21 & 70 & -84 \\
$\tau_{8}=15$ & -28 & 126 & -270 \\
\hline
\end{tabular}

and let's give how to find coefficients with the algorithm below. Coefficient values of $p^{2}$ in odd values of $T_{k}=2 k-1 ; A_{k}$ are found

$$
\begin{aligned}
& A_{1}=0 \\
& A_{k}=(-1)^{\tau_{k}}\left[\tau_{k}-2\right], k=2 \\
& A_{k}=(-1)^{\tau_{k}}\left[\frac{A_{k-1}}{(-1)^{\tau_{k}}}+(k-1)\right], k=3,4,5, \ldots
\end{aligned}
$$

Coefficients of $p^{4}$ given as $B_{k}$ become,

$$
\begin{aligned}
& B_{1}=0, B_{2}=0 \\
& B_{k}=\left(\tau_{k}-4\right), k=3 \\
& B_{k}=\left(B_{k-1}+4\right), k=4 \\
& B_{k}=\frac{A_{k-1}}{(-1)^{\tau}}+B_{k-1}+\left[B_{k-1}-B_{k-2}\right], k=5,6, \ldots
\end{aligned}
$$

and similarly, coefficients of $p^{6} ; C_{k}$ are found as

$$
\begin{aligned}
& C_{1}=C_{2}=C_{3}=0 \\
& C_{k}=(-1)^{\tau_{k}}\left[\tau_{k}-6\right], k=4
\end{aligned}
$$




$$
\begin{aligned}
& C_{k}=(-1)^{\tau_{k}}\left[\frac{C_{k-1}}{(-1)^{\tau_{k}}}+6\right], k=5 \\
& C_{k}=(-1)^{\tau_{k}}\left[B_{k-1}+\frac{C_{k-1}}{(-1)^{\tau_{k}}}+\left(\frac{C_{k-1}}{(-1)^{\tau_{k}}}-\frac{C_{k-2}}{(-1)^{\tau_{k}}}\right)\right], k=6,7, \ldots
\end{aligned}
$$

and also, considering statements in the left side of the inequalities, depending of coefficients of the terms $p^{i}, \quad i=3,5,7$ it is in the form of

Table 2

\begin{tabular}{lccc}
\hline$\tau_{k}=\tau$ & $p^{3}$ & $p^{5}$ & $p^{7}$ \\
\hline$\tau_{2}=3$ & 1 & & \\
$\tau_{3}=5$ & -4 & 1 & \\
$\tau_{4}=7$ & 10 & -6 & 1 \\
$\tau_{5}=9$ & -20 & 21 & -8 \\
$\tau_{6}=11$ & 35 & -56 & 36 \\
$\tau_{7}=13$ & -56 & 126 & -120 \\
$\tau_{8}=15$ & 84 & -252 & 330
\end{tabular}

The algorithm associated with coefficients of the terms $p^{3} ; Y_{k}$ is found as,

$$
\begin{aligned}
& Y_{1}=0 \\
& Y_{k}=\tau_{k}-2, k=2 \\
& Y_{k}=(-1)^{\tau_{k}}\left[Y_{k-1}+3\right], k=3 \\
& Y_{k}=\left[\frac{Y_{k-1}}{(-1)^{\tau_{k}}}-Y_{k-2}\right]+(k-1)+\frac{Y_{k-1}}{(-1)^{\tau_{k}}}, k=4,6,8, \ldots \\
& Y_{k}=(-1)^{\tau_{k}}\left[\left(Y_{k-1}-\frac{Y_{k-2}}{(-1)^{\tau_{k}}}\right)+(k-1)+Y_{k-1}\right], k=5,7,9, \ldots
\end{aligned}
$$

and the algorithm depending on this as being coefficient of $p^{5} ; N_{k}$ becomes,

$$
\begin{aligned}
& N_{1}=N_{2}=0 \\
& N_{k}=\tau_{k}-4, k=3 \\
& N_{k}=(-1)^{\tau_{k}}\left[N_{k-1}+5\right], k=4
\end{aligned}
$$

$$
\begin{aligned}
& N_{k}=Y_{k-1}+\frac{N_{k-1}}{(-1)^{\tau_{k}}}+\left[\frac{N_{k-1}}{(-1)^{\tau_{k}}}-N_{k-2}\right], k=5,7,9, \ldots \\
& N_{k}=(-1)^{\tau_{k}}\left[\frac{Y_{k-1}}{(-1)^{\tau_{k}}}+N_{k-1}+\left(N_{k-1}-\frac{N_{k-2}}{(-1)^{\tau_{k}}}\right)\right], k=6,8,10, \ldots
\end{aligned}
$$

Similarly, coefficient of $p^{7} ; L_{k}$ is in the form of

$$
\begin{aligned}
& L_{1}=L_{2}=L_{3}=0 \\
& L_{k}=\tau_{k}-6, k=4 \\
& L_{k}=(-1)^{\tau_{k}}\left[L_{k-1}+7\right], k=5 \\
& L_{k}=N_{k-1}+\frac{L_{k-1}}{(-1)^{\tau_{k}}}+\left(\frac{L_{k-1}}{(-1)^{\tau_{k}}}-L_{k-2}\right), k=6,8,10, \ldots \\
& L_{k}=(-1)^{\tau_{k}}\left[\frac{N_{k-1}}{(-1)^{\tau_{k}}}+L_{k-1}+\left(L_{k-1}-\frac{L_{k-2}}{(-1)^{\tau_{k}}}\right)\right], k=7,9,11, \ldots
\end{aligned}
$$

So, we get the following general form for stability conditions of the polynomial $G_{1}(\lambda)=\lambda^{2 k}-\lambda^{2 k-1}-p, \quad k=1,2, \vdots \quad$ are obtained with $p<0$ as

$\left|L_{k} p^{7}+N_{k} p^{5}+Y_{k} p^{3}+(-1)^{k+1} k p\right|<1+A_{k} p^{2}+B_{k} p^{4}+C_{k} p^{6}$

such that $p^{i}, i>0$. It is possible to get stability conditions for odd values of $K$ in the considered non-linear difference equation by using the algorithm above.

\subsection{Stability analysis of the equation (1) for even number values of $K$}

As per Theorem 2, each stability condition at $k$ steps should also added by the cases of positive absolute values of stability conditions from $k=1$ to that step. Similarly, if stability conditions for the values of $K=2 k, \quad k=1,2,3, \ldots$ in the Eq.(1) and corresponding characteristic polynomials of $\lambda^{2 k+1}-\lambda^{2 k}-p$ of linearized equation are calculated by using the condition iii) from Theorem 2, as follows:

for $k=1$,

$$
\lambda^{3}-\lambda^{2}-p, \quad|p|<1-p^{2}
$$

for $k=2$,

$$
\lambda^{5}-\lambda^{4}-p, \quad\left|p^{3}-2 p\right|<1-3 p^{2}+p^{4}
$$


for $k=3$,

$$
\lambda^{7}-\lambda^{6}-p, \quad\left|p^{5}-4 p^{3}+3 p\right|<1-6 p^{2}+5 p^{4}-p^{6}
$$

for $k=4$,

$$
\lambda^{9}-\lambda^{8}-p,\left|p^{7}-6 p^{5}+10 p^{3}-4 p\right|<1-10 p^{2}+15 p^{4}-7 p^{6}+p^{8}
$$

are obtained. It is seen that coefficients of $p^{i}$, $i=2,4,6 \ldots, 2 k$ which are on the right side of the inequality, are in the form of

Table 3

\begin{tabular}{llll}
\hline$\tau_{k}=\tau$ & $p^{2}$ & $p^{4}$ & $p^{6}$ \\
\hline$\tau_{1}=2$ & -1 & & \\
$\tau_{2}=4$ & -3 & 1 & \\
$\tau_{3}=6$ & -6 & 5 & -1 \\
$\tau_{4}=8$ & -10 & 15 & -7 \\
$\tau_{5}=10$ & -15 & 35 & -28 \\
$\tau_{6}=12$ & -21 & 70 & -84 \\
$\tau_{7}=14$ & -28 & 126 & -270
\end{tabular}

and let's give how to find coefficients with the algorithm below. Coefficients of $p^{2} ; M_{i}$ are calculated with

$$
\begin{aligned}
& M_{k}=(-1)^{\tau_{k}-1}\left[\tau_{k}-1\right], k=1 \\
& M_{k}=(-1)^{\tau_{k}-1}\left[\frac{M_{k-1}}{(-1)^{\tau_{k}-1}}+2\right], k=2 \\
& M_{k}=(-1)^{\tau_{k}-1}\left[\frac{M_{k-1}}{(-1)^{\tau_{k}-1}}+k\right], k=3,4,5, \ldots
\end{aligned}
$$

Coefficients of $p^{4} ; P_{k}$ are in the form of

$$
\begin{aligned}
& P_{1}=0 \\
& P_{k}=\tau_{k}-3, k=2 \\
& P_{k}=P_{k-1}+4, k=3 \\
& P_{k}=\frac{M_{k-1}}{(-1)^{\tau_{k}-1}}+P_{k-1}+\left[P_{k-1}-P_{k-2}\right], i=4,5,6, \ldots
\end{aligned}
$$

For coefficients of $p^{6} ; S_{i}$, it can be written that

$$
\begin{aligned}
& S_{1}=S_{2}=0 \\
& S_{k}=(-1)^{\tau_{k}-1}\left[T_{k}-5\right], k=3 \\
& S_{k}=(-1)^{\tau_{k}-1}\left[\frac{S_{k-1}}{(-1)^{\tau_{k}-1}}+6\right], k=4 \\
& S_{k}=(-1)^{\tau_{k}-1}\left[P_{k-1}+\frac{S_{k-1}}{(-1)^{\tau_{k}-1}}+\left(\frac{S_{k-1}}{(-1)^{\tau_{k}-1}}-\frac{S_{k-2}}{(-1)^{\tau_{k}-1}}\right)\right], k=5,6,7, \ldots
\end{aligned}
$$

and it is seen that coefficients of the terms $p^{i}$, $i=3,5,7$ which are on the left side of the inequality, become

Table 4

\begin{tabular}{lccc}
\hline$\tau_{k}=\tau$ & $p^{3}$ & $p^{5}$ & $p^{7}$ \\
\hline$\tau_{2}=4$ & 1 & & \\
$\tau_{3}=6$ & -4 & 1 & \\
$\tau_{4}=8$ & 10 & -6 & 1 \\
$\tau_{5}=10$ & -20 & 21 & -8 \\
$\tau_{6}=12$ & 35 & -56 & 36 \\
$\tau_{7}=14$ & -56 & 126 & -120
\end{tabular}

Depending on these coefficients, while coefficient of $p^{3} ; R_{k}$ is calculated with the algorithm of

$$
\begin{aligned}
& R_{1}=0 \\
& R_{k}=\left[\tau_{k}-3\right], k=2 \\
& R_{k}=(-1)^{\tau_{k}-1}\left[R_{k-1}+3\right], k=3 \\
& R_{k}=\left[\frac{R_{k-1}}{(-1)^{\tau_{k}-1}}-R_{k-2}\right]+(k-1)+\frac{R_{k-1}}{(-1)^{\tau_{k}-1}}, k=4,6,8, \ldots \\
& R_{k}=(-1)^{\tau_{k}-1}\left[\left(R_{k-1}-\frac{R_{k-2}}{(-1)^{\tau_{k}-1}}\right)+(k-1)+R_{k-1}\right], k=5,7,9, \ldots
\end{aligned}
$$

coefficient of the term $p^{5} ; V_{k}$ is in the form of

$$
\begin{aligned}
& V_{1}=V_{2}=0 \\
& V_{k}=\left[\tau_{k}-5\right], k=3 \\
& V_{k}=(-1)^{\tau_{k}-1}\left[V_{k-1}+5\right], k=4
\end{aligned}
$$




$$
\begin{aligned}
& V_{k}=\left[R_{k-1}+\frac{V_{k-1}}{(-1)^{\tau_{k}-1}}\right]+\left[\frac{V_{k-1}}{(-1)^{\tau_{k}-1}}-V_{k-2}\right], k=5,7,9, . . \\
& V_{k}=(-1)^{\tau_{k}-1}\left[\left(\frac{R_{k-1}}{(-1)^{\tau_{k}-1}}+V_{k-1}\right)+\left(V_{k-1}-\frac{V_{k-2}}{(-1)^{\tau_{k}-1}}\right)\right], k=6,8,10, \ldots
\end{aligned}
$$

Again, coefficient of the term $p^{7} ; Z_{k}$ becomes

$$
\begin{aligned}
& Z_{1}=Z_{2}=Z_{3}=0 \\
& Z_{k}=\tau_{k}-7, k=4 \\
& Z_{k}=(-1)^{\tau_{k}-1}\left[Z_{k}+7\right], k=5 \\
& Z_{k}=\left(V_{k-1}+\frac{Z_{k-1}}{(-1)^{\tau_{k}-1}}\right)+\left(\frac{Z_{k-1}}{(-1)^{\tau_{k}-1}}-Z_{k-2}\right), k=6,8,10, \ldots \\
& Z_{k}=(-1)^{\tau_{k}-1}\left[\left(V_{k-1}+\frac{Z_{k-1}}{(-1)^{\tau_{k}-1}}\right)+\left(\frac{Z_{k-1}}{(-1)^{\tau_{k}-1}}-Z_{k-2}\right)\right], k=7,9,11, \ldots
\end{aligned}
$$

So, we get the following general form for stability conditions of the polynomial $G_{2}(\lambda)=\lambda^{2 k+1}-\lambda^{2 k}-p, \quad k=1,2,3,4 \quad$ are obtained with $p>-2$ as

$$
\left|Z_{k} p^{7}+V_{k} p^{5}+R_{k} p^{3}+(-1)^{k+1} k p\right|<1+M_{k} p^{2}+P_{k} p^{4}+S_{k} p^{6}+\ldots+p^{2 k}
$$

It is possible to get stability conditions for even values of $K$ in the considered non-linear difference equation by using the algorithm above.

\section{Examples}

In order to support our theoretical result, we consider some numerical examples in this section.

Example 4 Let us consider Eq.(1) for the case $\tau=1$ studied [see Çelik et al (2008)]. Then, we have

$$
N_{t+1}=\mu N_{t} f\left(N_{t-1}\right) \text {. }
$$

From Definition 1, we obtain

$$
\mu f\left(N^{*}\right)=1 \text {. }
$$

If $Y_{t+1}=N_{t}=f_{2}\left(N_{t}, Y_{t}\right)$ is taken, then we get $N_{t+1}=\mu N_{t} f\left(N_{t-1}\right)=\mu N_{t} f\left(Y_{t}\right)=f_{1}\left(N_{t}, Y_{t}\right)$. So, the Jacobian matrix evaluated about $N^{*}$ is

$$
J=\left(\begin{array}{cc}
\mu f\left(N^{*}\right) & \mu N^{*} f^{\prime}\left(N^{*}\right) \\
1 & 0
\end{array}\right)
$$

so that its characteristic polynomial is

$$
G_{1}(\lambda)=\lambda^{2}-\lambda-\mu N^{*} f^{\prime}\left(N^{*}\right) .
$$

Note that $K=1$. From the condition (i)-(ii) in Theorem 2, we can write

$$
p<0 \text {, }
$$

and if the inequality (11) is used instead of the condition (iii) in Theorem 2, then we have

$$
|p|<1 \text {. }
$$

such that $A_{1}=B_{1}=C_{1}=Y_{1}=N_{1}=L_{1}=0$. The result is confirmed.

Example 5 Let us consider Eq.(1) for the case $\tau=2$ studied [see Merdan and Ak Gümüş (2012)]. Then, we have

$$
N_{t+1}=\mu N_{t} f\left(N_{t-2}\right) .
$$

Smilarly; let us choose the following

$$
\begin{aligned}
& Z_{t+1}=Y_{t}=f_{3}\left(N_{t}, Y_{t}\right) \\
& Y_{t+1}=N_{t}=f_{2}\left(N_{t}, Y_{t}\right) \\
& N_{t+1}=\mu N_{t} f\left(Z_{t}\right)=f_{1}\left(N_{t}, Y_{t}\right) .
\end{aligned}
$$

So, the Jacobian matrix can be written as

$$
J=\left(\begin{array}{ccc}
\mu f\left(N^{*}\right) & 0 & \mu N^{*} f^{\prime}\left(N^{*}\right) \\
1 & 0 & 0 \\
0 & 1 & 0
\end{array}\right)
$$

whose characteristic polynomial is

$$
G_{2}(\lambda)=\lambda^{3}-\lambda^{2}-\mu N^{*} f^{\prime}\left(N^{*}\right) \text {. }
$$

Note that $K=2$. From the condition (i)-(ii) in Theorem 2, we can write

$$
p>-2 \text {, }
$$

and if the inequality (16) is used instead of the condition (iii) in Theorem 2, then we have

$$
|p|<1-p^{2} \text {. }
$$

such that $P_{1}=S_{1}=R_{1}=V_{1}=Z_{1}=0$ and $M_{1}=-1$ э $M_{1}=(-1)^{\tau_{1}-1}\left[\tau_{1}-1\right], \quad \tau_{1}=2$. The result is confirmed.

\section{Conclusion}

The importance of stability study is great in population 
dynamics. Thanks to this, whether the population has reached an equilibrium is detected. And this provides us information about what the number of that population will be in the future.

In this study, stability generalization of dynamic model, which is based on the Schur-Cohn criterion, is provided with a new method to a certain step. Also, it can be seen that the condition iii) in Schur-Cohn criteria can be obtained more easily.

\section{References}

Merdan, H., Ak Gümüş, Ö., 2012. Stability analysis of a general discrete-time population model involving delay and Allee effect, Applied Mathematics and Computation., 219, 1821-1832.

Çelik, C., Merdan, H., Duman, O. and Akın, Ö., 2008. Allee effects on population dynamics with delay, Chaos, Solutions and Fractals, 37, 65-74.

Elaydi S. N., 2006. An Introduction to Difference Equations, New York.

Merdan, H., Karaoğlu, E., 2012. Consequences of Allee Effects on stability analysis of the population model $x_{t+1}=\lambda x_{t} f\left(x_{t-3}\right)$, Hacettepe Journal of Mathematics and Statistics, 41, (5), $751-765$.

Allen, L.J.S., 2007. An Introduction to Mathematical Biology, Pearson, New Jersey.

Ak Gümüş, Ö., 2014. Global and local stability analysis in a nonlinear discrete-time population model, Advances in Difference Equations, 299, 1687-1847.

Ak Gümüş, Ö., Köse, H., 2012. On the stability of delay population dynamics related with Allee effects, Mathematical and Computational Applications, 17, (1), 56-67.

Ak Gümüş, Ö., Köse, H., 2012. Allee effect on a new delay population model and stability analysis, Journal of Pure and Applied Mathematics: Advances and Applications, 7, 21-31. 